\title{
Fecal Microbiota Transplantation for Fibromyalgia: A Case Report and Review of the Literature
}

\author{
T. Thurm1, J. N. Ablin², D. Buskila" ${ }^{3,4}$, N. Maharshak ${ }^{*}$ \\ ${ }^{1}$ The Bacteriotherapy Clinic, Department of Gastroenterology and Liver Diseases, Tel Aviv Sourasky Medical Center, Affiliated to \\ the Sackler Faculty of Medicine, Tel Aviv University, Tel Aviv, Israel \\ ${ }^{2}$ Institute of Rheumatology, Tel Aviv Sourasky Medical Center, and Sackler Faculty of Medicine, Tel Aviv University, Tel Aviv, \\ Israel \\ ${ }^{3}$ Department of Medicine H, Soroka Medical Center, Beer Sheva, Israel \\ ${ }^{4}$ Faculty of Health Sciences, Ben-Gurion University of the Negev, Beer Sheva, Israel \\ Email: *nitsanm@tlvmc.gov.il
}

How to cite this paper: Thurm, T., Ablin, J.N., Buskila, D. and Maharshak, N. (2017) Fecal Microbiota Transplantation for Fibromyalgia: A Case Report and Review of the Literature. Open Journal of Gastroenterology, 7, 131-139.

https://doi.org/10.4236/ojgas.2017.74015

Received: February 25, 2017

Accepted: April 25, 2017

Published: April 28, 2017

Copyright () 2017 by authors and Scientific Research Publishing Inc. This work is licensed under the Creative Commons Attribution International License (CC BY 4.0).

http://creativecommons.org/licenses/by/4.0/

\begin{abstract}
A 58-year-old patient diagnosed with fibromyalgia, irritable bowel syndrome (IBS) and chronic fatigue syndrome (CFS), non-responsive to variety of treatments over the years, suffered from significant social and occupational disabilities. The patient was interested in fecal microbiota transplantation (FMT), but given that FMT is not approved for these indications, he used an online protocol for FMT screening and preparation and self-instilled the filtrate using an enema 6 times. FMT resulted in a gradual improvement of symptoms and 9 months after the last treatment, the patient reported full recovery of symptoms, going back to work at full time employment. Improvement of symptoms was associated with major alterations of the enteric microbiota, according to next generation sequencing analysis performed before the first FMT and after the last FMT. Most prominent alterations at the genus level included a decrease in fecal Streptococcus proportion from $26.39 \%$ to $0.15 \%$ and an increase in Bifidobacterium from $0 \%$ to $5.23 \%$. This case is added to several additional case reports that demonstrated the effectivity of FMT in these functional disorders that are lacking an otherwise good medical therapeutic intervention. We conclude that randomized controlled trials are required to ground FMT as a possible therapy for these difficult-to-treat conditions.
\end{abstract}

\section{Keywords}

Microbiota, Microbiome, Irritable Bowel Syndrome, Functional Disorders, Dysbiosis, Chronic Fatigue Syndrome 


\section{Case Presentation}

A 58-year-old patient presented to our outpatient clinic complaining of severe diffuse pain, insomnia, diarrhea, abdominal pain, bloating, photophobia, hypersensitivity to odor, noise, light touch, tinnitus and palpitations. He suffered from cognitive impairment: memory loss, concentration deficit and depression with suicidal thoughts.

Most of his symptoms started 18 years earlier at the age of 40 . He was diagnosed with fibromyalgia, IBS and CFS. On his physical examination, typical tender points for fibromyalgia were accessed. He underwent lactose, glucose, fructose and sorbitol breath tests, stool cultures, serological testing for inflammatory bowel disease and celiac, fecal calprotectin level test, upper endoscopy and colonoscopy. As all 4 breath tests were positive, the patient was diagnosed with small intestinal bacterial overgrowth (SIBO). ASCA IgA was also mildly positive and he underwent a video capsule endoscopy that was normal.

Throughout the years, the patient was treated with selective serotonin reuptake inhibitors (SSRIs), serotonin antagonist and reuptake inhibitors (SARIs), serotonin and norepinephrine reuptake inhibitors (SNRIs), tricyclic antidepressants (TCAs), gamma-aminobutyric acid (GABA), pregabalin, tramadol, Omega 3, Cognitive Behavioral Therapy (CBT), medical cannabis, St. John's wort (Hypericum perforatum), acupuncture, medicinal hot springs, hydrotherapy, mild exercise, all without considerable effect.

After a personal tragedy, the disease progressed and the patient became disabled and stopped working. He spent most of his days in bed, unable to perform minimal physical activity; his insomnia has worsened despite treatment with melatonin and sleeping pills. Memory impairment had become severe, with memory loss of close relatives and friends, and marked disorientation. Social security has granted the patient $70 \%$ general disability and a complete (100\%) occupational disability.

The patient sent his own stool samples for microbial next generation sequencing analysis at a commercial lab. The results demonstrated high levels of Streptococci (Table 1). Stool culture, done at the same time, was positive for Candida. He was treated with Hydroxychloroquine and Rifampicin, VSL\#3 (probiotic), dietary change to low carbohydrates and low sugar diet, reporting mild and short standing improvement.

The patient was interested in FMT as an experimental treatment for his mixed symptoms caused by fibromyalgia, CFS and IBS. Given that this type of treatment is not approved for these indications, he used an online protocol for FMT screening and preparation. His son was screened for HIV, HCV, fecal parasites and bacterial cultures. Stool was homogenized with a food processor and was self-instilled using an enema. Within 24 hours he experienced dramatic improvement of symptoms that lasted for 6 weeks. Four consecutive FMTs resulted with the same transient improvement of symptoms, lasting for approximately 6 weeks each. The improvement from the sixth course lasted for over 9 months and included additional treatment for SIBO with FODMAP and rifaximin. 
Table 1. Fecal microbiota analysis performed prior and post fecal microbial transplantation (FMT). A 16S rDNA genome sequencing demonstrating microbial composition alterations at the "Genus level" following FMT.

\begin{tabular}{|c|c|c|c|c|}
\hline Phylum & Family & Genus & $\begin{array}{l}\% \text { of total } \\
\text { (Pre FMT) }\end{array}$ & $\begin{array}{c}\% \text { of total } \\
\text { (Post FMT) }\end{array}$ \\
\hline \multirow{36}{*}{$\begin{array}{c}\text { Firmicutes } \\
(\text { gram }+)\end{array}$} & Lachnospiraceae & Anaerostipes & $7.92^{*}$ & 0.09 \\
\hline & & Coprococcus & 0.72 & 1.66 \\
\hline & & Dorea & 6.85 & 0.61 \\
\hline & & Moryella & 0 & 0 \\
\hline & & Roseburia & 32.11 & 3.01 \\
\hline & & Sporobacterium & 0 & 0 \\
\hline & & Syntrophococcus & 0 & 0 \\
\hline & Ruminococcaceae & Acetanaerobacterium & 0 & 0 \\
\hline & & Acetivibrio & $1.22^{*}$ & 0 \\
\hline & & Ethanoligenens & 0 & 0 \\
\hline & & Faecalibacterium & 0.08 & 5.65 \\
\hline & & Papillibacter & 0 & 0 \\
\hline & & Ruminococcus & $1.27^{\star}$ & 4.51 \\
\hline & & Sporobacterium & 0 & 0 \\
\hline & & Subdoligranulum & 0.17 & 0 \\
\hline & Clostridiaceae & Butyricicoccus & 0 & 0 \\
\hline & & Clostridium & 0 & 1.58 \\
\hline & & Lactonifactor & $0.08^{*}$ & 0 \\
\hline & Eubacteriaceae & Naaerofustis & 0 & 0 \\
\hline & & Eubacterium & 0 & $4.91^{*}$ \\
\hline & Blautia & Blautia & 21.51 & 10.92 \\
\hline & Howardella & Howardella & 0 & 0 \\
\hline & Lactobacillaceae & Lactobacillus & 0.2 & 0.25 \\
\hline & Enterococcaceae & Enterococcus & 0 & $0.03^{*}$ \\
\hline & Streptococcaceae & Lactococcus & 0 & 0 \\
\hline & & Streptococcus & $26.39^{*}$ & 0.15 \\
\hline & Leuconostocaceae & Leuconostoc & 0 & 0.01 \\
\hline & Erysipelotrichaceae & Catenibacterium & 0 & 0 \\
\hline & & Coprobacillus & 0.54 & 0.5 \\
\hline & & Holdemania & 0 & 0 \\
\hline & & Turicibacter & $0^{*}$ & $0.04^{*}$ \\
\hline & Veillonellaceae & Dialistr & 0 & 0 \\
\hline & & Megamonas & 0 & 0 \\
\hline & & Megasphaera & 0 & $2.26^{*}$ \\
\hline & Oscillospiaceae & Oscillibacter & 0.29 & 0 \\
\hline & Staphlococcaceae & Staphylococcus & 0 & 0 \\
\hline \multirow[t]{5}{*}{$\begin{array}{l}\text { Bacteroidetes } \\
\text { (gram-) }\end{array}$} & Bacteroidaceae & Bacteroides & 0.42 & $21.11^{*}$ \\
\hline & Rikenellaceae & Alistipes & 0 & 0 \\
\hline & Porphyromonadaceae & Barnesiella & 0 & 0 \\
\hline & & Odoribacter & 0 & 0.04 \\
\hline & & Parabacteroides & 0 & 1.04 \\
\hline
\end{tabular}




\begin{tabular}{ccccc} 
Continued & & & & \\
\hline & Prevotellaceae & Prevotella & 0 & $17.64^{*}$ \\
& & Xylanibacter & 0 & 0 \\
\hline $\begin{array}{c}\text { Actinobacteria } \\
\text { (gram+) }\end{array}$ & Bifidobacteriaceae & Bifidobacterium & $0^{*}$ & 5.23 \\
& Actinomycetaceae & Actinomyces & 0 & 0.01 \\
& Micrococcineae & Rothia & 0 & 0 \\
& Coriobacteriaceae & Asaccharobacter & $0^{*}$ & $0^{*}$ \\
& & Collinsella & 0 & 6.73 \\
& & Olsenella & 0 & 0 \\
\hline Proteobacteria & Slackia & 0 & $1.47^{*}$ \\
\cline { 3 - 3 }-$)$ & Enterobacteriaceae & Escherichia/Shigella & 0 & 0.45 \\
& & Klebsiella & 0 & 0 \\
& & Cutterella & 0 & 0.31 \\
& Desulfovibrionaceae & Lawsonia & 0 & 0 \\
\hline
\end{tabular}

*Abnormal \% of total microbiota as defined by the reference of the commercial lab.

The patient reported marked improvement with total resolution of fatigue and depression, marked improvement of insomnia, oversensitivity to touch, odor and noise. Cognitive impairment has also improved. A physical examination by a rheumatologist (JN A) was normal with no evidence of synovitis or tender fibromyalgia points, concluding that all his symptoms had improved. The patient returned to full employment and is now asymptomatic for over a year.

A second stool microbial analysis demonstrated significant changes compared to the first analysis (Table 1, Table 2). Most marked was a decrease in the proportion of the Firmicutes phylum from $99.35 \%$ to $36.17 \%$ and an increase in the Bacteriodetes phylum from $0.42 \%$ to $39.82 \%$ post FMT. At the genus level, fecal Streptococcus proportion fell from $26.39 \%$ to $0.15 \%$ and Bifidobacterium increased from $0 \%$ to $5.23 \%$. Additional changes included bacterial diversity index that was reduced from 3.21 to 2.55 post FMTs and a negative stool culture for Candida.

\section{Discussion}

Functional disorders, such as fibromyalgia, CFS and IBS, affect many patients and are frequently associated. These disorders clinically differ with an unclear pathogenesis.

Fibromyalgia affects up to $2 \%$ of general population [1]. Pain is a predominant symptom of fibromyalgia. However, fatigue, non-refreshed sleep, mood disturbance and cognitive impairment are common, and have an important influence on quality of life [2]. The European league against Rheumatism (EULAR) recommendations, for the management of fibromyalgia were published in 2016: Graduated approach aiming at improving health-related quality of life by non-pharmacological modalities is advised as first line therapy [3].

CFS is diagnosed after at least 6 months of unexplained fatigue [4]. CBT and graded exercise therapy (GET) have shown moderate effectiveness [5]. 
Table 2. Fecal microbiota analysis performed prior and post fecal microbial transplantation (FMT). A 16S rDNA genome sequencing demonstrating microbial composition alterations at the "Phylum" and "Family" level following FMT.

\begin{tabular}{ccc}
\hline & \% Pre FMT & \% Post FMT \\
\hline Firmicutes & 99.35 & 36.17 \\
Lachnospiraceae & 47.6 & 5.37 \\
Ruminococcaceae & 2.74 & 10.16 \\
Clostridiaceae & 0.08 & 1.58 \\
Bacteroidetes & 0.42 & 39.82 \\
Actinobacteria & 0 & 13.44 \\
Proteobacteria & 0 & 0.76 \\
\hline
\end{tabular}

IBS is a functional disorder characterized by abdominal pain or discomfort associated with defecation or change in bowel habits according the Rome criteria [6]. IBS usually causes long-term symptoms, which may occur in episodes. The symptoms interfere with daily life and social functioning in many patients. The prevalence of IBS in Europe and North America is estimated to be $10 \%$ to 15\% [7].

There is a marked overlap between these conditions. The prevalent theory suggests that CNS dysfunction of unknown origin causes over responsiveness to sensory perception stimuli that are within the normal range among healthy individuals in patients suffering from fibromyalgia, CFS, IBS and other functional disorders [8]. Investigations using standardized criteria reported $42 \%-70 \%$ of fibromyalgia patients meeting IBS criteria [9] [10] and one study demonstrated $92 \%$ IBS in patients diagnosed with CFS [11]. Many patients report being diagnosed with fibromyalgia and later the diagnosis is converted to CFS and vice versa.

During the last decade, the importance of the enteric microbiome for health and disease conditions has emerged. Changes in the composition of gut microbiota (dysbiosis), have been associated with gastrointestinal, metabolic, autoimmune, allergic and neuropsychiatric disorders [12] and specifically in IBS patients [13] [14], fibromyalgia [14] and in CFS [15]. Moreover, interventions altering the enteric microbiota, such as probiotics therapy [16], and rifaximin [17] have shown efficacy in IBS patients.

FMT, which is the infusion of liquid filtrate feces from a healthy donor, into the gut of an affected recipient, has shown significant benefit for the treatment of Clostridium difficile infection (CDI) [18] [19] and is currently the recommended therapy for recurrent CDI [20]. FMT has also shown positive results for the treatment of inflammatory bowel disease [21] in multiple studies and have demonstrated some efficacy in non GI disorders, such as in Parkinson's disease [22], myoclonus dystonia [23], multiple sclerosis [24], obesity, insulin resistance and metabolic syndrome [25]. In a study of 60 CFS patients treated with FMT, response rate was $70 \%$. Twelve of the patients were contacted after 15 - 20 years, 7 reported full recovery and 5 reported no CFS symptoms for 1.5 and 3 years 
following FMT [26].

In IBS patients, few studies, mostly case reports and case series, have demonstrated positive results for FMT [27] and it is currently tested in randomized controlled trials [NIH. clinical trialNCT02328547].

D-lactic acid producing Enterococcus and Streptococcus species were shown to be over-represented in CFS patients [28]. A recent study comparing microbiota of CFS patient to controls, observed significantly lower levels of Faecalibacterium and Bifidobacterium in the CFS population compared to healthy controls [15]. Faecalibacterium is known to have anti-inflammatory properties [29] and Bifidobacterium, commonly used as probiotics [30], was previously reported to reduce CRP levels in a cohort of CFS patients [31]. In addition, a significantly higher load of Candida albicans was found in CFS patients when in the acute phase of illness compared with when in remission [32]. Although the clinical significance of these findings is yet to be determined and although the fecal microbial analysis was not performed as a part of a clinical trial, some of the microbial alterations cited above are in line with the microbial alterations observed before and after FMT in our case. Specifically, the decrease of fecal Streptococcus and increased Bifidobacterium proportions and loss of fecal Candida, may support the beneficial properties of FMT.

Nevertheless, our results should be interpreted with caution, given that in addition to self FMTs, the patient underwent multiple additional interventions such as different dietary regiments, therapeutic trials with multiple medications, antibiotics and probiotics. These can also be responsible, at least partially, for the microbial alterations that were found, such as the decreased microbial diversity index that dropped from 3.21 to 2.55 perhaps due to antibiotics treatment.

Moreover, as in any treatment modality implemented for chronic pain, a significant placebo response must also be taken under consideration. However, the clinical improvement was clearly associated with the FMTs treatments.

No RCT's or case series have been published on FMT for fibromyalgia. To our knowledge, this is the first case of self-executed FMT resulting in full recovery of fibromyalgia and CFS. In the absence of satisfactory cognitive or pharmacological treatment for these disorders, few publications reported the experience of FMT for these indications.

Although there are no randomized control trials (RCTs) supporting this treatment, in a few case series on CFS and reports on fibromyalgia, FMT induced long term remission of CFS [26] and could be beneficial for fibromyalgia. This is a low risk procedure with mostly mild and short term complications [33] [34], with possible long term remission that may result in a significant cost effectiveness. A major concern is that the fame of FMT may lead patients to use a "do-it-yourself" approach (as in this case), without medical supervision, with possibly harmful consequences [35] [36]. A case of UC patient who suffered cytomegalovirus infection after performing FMT without donor screening was reported [37] and therefore we do not support performing FMT without appropriate medical care. 


\section{Conclusions}

We have described a case of self-performed FMT, in which FMT was extremely beneficial in a severely ill patient who suffered from a combination of three functional disorders-fibromyalgia, CFS and IBS. The patient had high levels of Candida and Streptococci that have normalized after the FMT.

We conclude that FMT should be tested as a possible treatment for fibromyalgia and CFS. Controlled data regarding the microbiome characteristics of patients suffering from fibromyalgia should be obtained, and RCTs are required to ground FMT as a possible therapy for these difficult-to-treat conditions, to learn whether this approach is truly beneficial, who is the right target population, who should serve as donors and what is the right protocol of performing FMT for this patient population: number of FMTs, volume and optimal way of administration.

\section{References}

[1] Queiroz, L.P. (2013) Worldwide Epidemiology of Fibromyalgia. Current Pain and Headache Reports, 17, 356.

[2] Fietta, P. and Manganelli, P. (2007) Fibromyalgia and Psychiatric Disorders. Acta Biomed, 78, 88-95.

[3] Macfarlane, G.J., et al. (2016) EULAR Revised Recommendations for the Management of Fibromyalgia. Annals of the Rheumatic Diseases, 76, 318-328.

[4] Rimbaut, S., Van Gutte, C., Van Brabander, L. and Vanden Bossche, L. (2016) Chronic Fatigue Syndrome-An Update. Acta Clinica Belgica, 71, 273-280.

[5] Harvey, S.B. and Wessely, S. (2009) Chronic Fatigue Syndrome: Identifying Zebras amongst the Horses. BMC Medicine, 7, 58.

[6] Mearin, F., et al. (2016) Bowel Disorders. Gastroenterology, 150, 1393-1407.e5.

[7] Quigley, E.M., et al. (2016) World Gastroenterology Organisation Global Guidelines Irritable Bowel Syndrome: A Global Perspective Update September 2015. Journal of Clinical Gastroenterology, 50, 704-713. https://doi.org/10.1097/MCG.0000000000000653

[8] Albrecht, P.J. and Rice, F.L. (2016) Fibromyalgia Syndrome Pathology and Environmental Influences on Afflictions with Medically Unexplained Symptoms. Reviews on Environmental Health, 31, 281-294. https://doi.org/10.1515/reveh-2015-0040

[9] Veale, D., et al. (1991) Primary Fibromyalgia and the Irritable Bowel Syndrome: Different Expressions of a Common Pathogenetic Process. British Journal of Rheumatology, 30, 220-222. https://doi.org/10.1093/rheumatology/30.3.220

[10] Sivri, A., et al. (1996) Bowel Dysfunction and Irritable Bowel Syndrome in Fibromyalgia Patients. Clinical Rheumatology, 15, 283-286.

https://doi.org/10.1007/BF02229708

[11] Aaron, L.A., Burke, M.M. and Buchwald, D. (2000) Overlapping Conditions among Patients with Chronic Fatigue Syndrome, Fibromyalgia, and Temporomandibular Disorder. Archives of Internal Medicine, 160, 221-227. https://doi.org/10.1001/archinte.160.2.221

[12] Xu, M.Q., et al. (2015) Fecal Microbiota Transplantation Broadening Its Application beyond Intestinal Disorders. World Journal of Gastroenterology, 21, 102-111. https://doi.org/10.3748/wjg.v21.i1.102 
[13] Nagel, R., et al. (2016) Comparison of Faecal Microbiota in Blastocystis-Positive and Blastocystis-Negative Irritable Bowel Syndrome Patients. Microbiome, 4, 47.

[14] Wallace, D.J. and Hallegua, D.S. (2004) Fibromyalgia: The Gastrointestinal Link. Current Pain and Headache Reports, 8, 364-368. https://doi.org/10.1007/s11916-996-0009-Z

[15] Giloteaux, L., et al. (2016) Reduced Diversity and Altered Composition of the Gut Microbiome in Individuals with Myalgic Encephalomyelitis/Chronic Fatigue Syndrome. Microbiome, 4, 30.

[16] Zhang, Y., et al. (2016) Effects of Probiotic Type, Dose and Treatment Duration on Irritable Bowel Syndrome Diagnosed by Rome III Criteria: A Meta-Analysis. BMC Gastroenterology, 16, 62.

[17] Lembo, A., et al. (2016) Repeat Treatment with Rifaximin Is Safe and Effective in Patients with Diarrhea-Predominant Irritable Bowel Syndrome. Gastroenterology, 151, 1113-1121.

[18] Youngster, I., et al. (2014) Fecal Microbiota Transplant for Relapsing Clostridium Difficile Infection Using a Frozen Inoculum from Unrelated Donors: A Randomized, Open-Label, Controlled Pilot Study. Clinical Infectious Diseases, 58, 1515 1522. https://doi.org/10.1093/cid/ciu135

[19] Debast, S.B., Bauer, M.P. and Kuijper, E.J. (2014) European Society of Clinical Microbiology and Infectious Diseases: Update of the Treatment Guidance Document for Clostridium Difficile Infection. Clinical Microbiology and Infection, 20, 1-26.

[20] Surawicz, C.M., et al. (2013) Guidelines for Diagnosis, Treatment, and Prevention of Clostridium Difficile Infections. The American Journal of Gastroenterology, 108, 478-498.

[21] Moayyedi, P., et al. (2015) Fecal Microbiota Transplantation Induces Remission in Patients with Active Ulcerative Colitis in a Randomized Controlled Trial. Gastroenterology, 149, 102-109.

[22] Ananthaswamy, A. (2011) Faecal Transplant Eases Symptoms of Parkinson's. New Scientist, 209, 8-9.

[23] Borody, T.J., Rosen, D.M., Torres, M., Campbell, J. and Nowak, A. (2011) Myoclonus-Dystonia (M-D) Mediated by GI Microbiota Diarrhoea Treatment Improves M-D Symptoms. The American Journal of Gastroenterology, 106, S352.

[24] Borody, T.J., Leis, L.S., Campbell, J., et al. (2011) Fecal Microbiota Transplantation (FMT) in Multiple Sclerosis (MS). The American Journal of Gastroenterology, 106, S352.

[25] Vrieze, A., et al. (2012) Transfer of Intestinal Microbiota from Lean Donors Increases Insulin Sensitivity in Individuals with Metabolic Syndrome. Gastroenterol$o g y, 143,913-916$.

[26] Borody, T.J., Nowak, A. and Finlayson, S. (2012) The GI Microbiome and Its Role in Chronic Fatigue Syndrome: A Summary of Bacteriotherapy. Journal of the Australasian College of Nutritional and Environmental Medicine, 31, 3-8.

[27] Pinn, D.M., Aroniadis, O.C. and Brandt, L.J. (2014) Is Fecal Microbiota Transplantation the Answer for Irritable Bowel Syndrome? A Single-Center Experience. The American Journal of Gastroenterology, 109, 1831-1832. https://doi.org/10.1038/ajg.2014.295

[28] Sheedy, J.R., et al. (2009) Increased d-Lactic Acid Intestinal Bacteria in Patients with Chronic Fatigue Syndrome. In Vivo, 23, 621-628.

[29] Sokol, H., et al. (2008) Faecalibacterium Prausnitzii Is an Anti-Inflammatory Commensal Bacterium Identified by Gut Microbiota Analysis of Crohn Disease Patients. 
Proceedings of the National Academy of Sciences, 105, 16731-16736. https://doi.org/10.1073/pnas.0804812105

[30] Furrie, E., et al. (2005) Synbiotic Therapy (Bifidobacterium Longum/Synergy 1) Initiates Resolution of Inflammation in Patients with Active Ulcerative Colitis: A Randomised Controlled Pilot Trial. Gut, 54, 242-249. https://doi.org/10.1136/gut.2004.044834

[31] Groeger, D., et al. (2013) Bifidobacterium Infantis 35624 Modulates Host Inflammatory Processes beyond the Gut. Gut Microbes, 4, 325-339. https://doi.org/10.4161/gmic.25487

[32] Evengard, B., et al. (2007) Increased Number of Candida Albicans in the Faecal Microflora of Chronic Fatigue Syndrome Patients during the Acute Phase of Illness. Scandinavian Journal of Gastroenterology, 42, 1514-1515. https://doi.org/10.1080/00365520701580397

[33] Wang, Z.K., et al. (2014) Intestinal Microbiota Pathogenesis and Fecal Microbiota Transplantation for Inflammatory Bowel Disease. World Journal of Gastroenterology, 20, 14805-14820. https://doi.org/10.3748/wjg.v20.i40.14805

[34] Kelly, C.R., et al. (2015) Update on Fecal Microbiota Transplantation 2015: Indications, Methodologies, Mechanisms, and Outlook. Gastroenterology, 149, 223-237. https://doi.org/10.1053/j.gastro.2015.05.008

[35] Choi, H.H. and Cho, Y.S. (2016) Fecal Microbiota Transplantation: Current Applications, Effectiveness, and Future Perspectives. Clinical Endoscopy, 49, 257-265. https://doi.org/10.5946/ce.2015.117

[36] El-Matary, W. (2013) Fecal Microbiota Transplantation: Long-Term Safety Issues. The American Journal of Gastroenterology, 108, 1537-1538. https://doi.org/10.1038/ajg.2013.208

[37] Hohmann, E.L., Ananthakrishnan, A.N. and Deshpande, V. (2014) Case Records of the Massachusetts General Hospital. Case 25-2014. A 37-Year-Old Man with Ulcerative Colitis and Bloody Diarrhea. The New England Journal of Medicine, 371, 668675. https://doi.org/10.1056/NEJMcpc1400842

Submit or recommend next manuscript to SCIRP and we will provide best service for you:

Accepting pre-submission inquiries through Email, Facebook, LinkedIn, Twitter, etc. A wide selection of journals (inclusive of 9 subjects, more than 200 journals)

Providing 24-hour high-quality service

User-friendly online submission system

Fair and swift peer-review system

Efficient typesetting and proofreading procedure

Display of the result of downloads and visits, as well as the number of cited articles

Maximum dissemination of your research work

Submit your manuscript at: http://papersubmission.scirp.org/

Or contact ojgas@scirp.org 